\title{
The Role of Hypoxia-Inducible Factor/Prolyl Hydroxylation Pathway in Deoxycorticosterone Acetate/Salt Hypertension in the Rat
}

\author{
Mohammad K Dallatu ${ }^{1}$, Elizabeth Nwokocha1 ${ }^{1}$, Ngozi AGU1 ${ }^{1}$, Choi Myung ${ }^{1}$, Mohammad A Newaz ${ }^{2}$, Gabriela Garcia ${ }^{3}$, Luan D Truong ${ }^{4}$ and \\ Adebayo O Oyekan ${ }^{\star *}$
}

${ }^{1}$ Center for Cardiovascular Diseases, Texas Southern University, USA

${ }^{2}$ College of Pharmacy, Chicago State University, USA

${ }^{3}$ Department of Medicine, Division of Renal Diseases and Hypertension, University of Colorado, USA

${ }^{4}$ Department of Pathology and Genomic Medicine, The Methodist Hospital, Houston, USA

\begin{abstract}
KKidney disease could result from hypertension and ischemia/hypoxia. Key mediators of cellular adaptation to hypoxia are oxygen-sensitive hypoxia inducible factor (HIF)s which are regulated by prolyl-4-hydroxylase domain (PHD)-containing dioxygenases. However, HIF activation can be protective as in ischemic death or promote renal fibrosis in chronic conditions. This study tested the hypothesis that increased HIF-1 $\alpha$ consequent to reduced PHD expression contributes to the attendant hypertension and target organ damage in deoxycorticosterone acetate (DOCA)/ salt hypertension and that PHD inhibition ameliorates this effect. In rats made hypertensive by DOCA/salt treatment (DOCA $50 \mathrm{mg} / \mathrm{kg} \mathrm{s} / \mathrm{c} ; 1 \% \mathrm{NaCl}$ orally), PHD inhibition with dimethyl oxallyl glycine (DMOG) markedly attenuated hypertension $(P<0.05)$, proteinuria $(P<0.05)$ and attendant tubular interstitial changes and glomerular damage $(P<0.05)$. Accompanying these changes, DMOG blunted the increased expression of kidney injury molecule $(K I M)-1(P<0.05)$, a marker of tubular injury and reversed the decreased expression of nephrin $(P<0.05)$, a marker of glomerular injury. DMOG also decreased collagen I staining $(P<0.05)$, increased serum nitrite $(P<0.05)$ and decreased serum 8-isopostane $(P<0.05)$. However, the increased HIF-1a expression $(P<0.01)$ and decreased PHD2 expression $(P<0.05)$ in DOCA/salt hypertensive rats was not affected by DMOG. These data suggest that reduced PHD2 expression with consequent increase in HIF-1 $\alpha$ expression probably results from hypoxia induced by DOCA/salt treatment with the continued hypoxia and reduced PHD2 expression evoking hypertensive renal injury and collagen deposition at later stages. Moreover, a PHD inhibitor exerted a protective effect in DOCA/salt hypertension by mechanisms involving increased nitric oxide production and reduced production of reactive oxygen species.
\end{abstract}

Keywords: Hypoxia inducible factor (HIF); Prolyl-4-hydroxylase domain (PHD); DOCA hypertension; KIM-1; ephrin; Collagen I; Nitric oxide; Reactive oxygen species

\section{Introduction}

Cardiovascular Diseases (CVDs) are intimately related to hypoxia and hypoxia inducible factor (HIF) is a key transcription factor that plays an important role in the cellular adaptive response to hypoxia such as glycolysis, erythrocytosis, and angiogenesis [1]. Expression of HIF$1 \alpha$ is negatively regulated by prolyl hydroxylase domain-containing protein (PHD), an enzyme that belongs to an evolutionarily conserved subfamily of dioxygenases which uses 2-oxogluatarate as substrate and $\mathrm{Fe}^{2+}$ and ascorbic acid as cofactors. PHD subfamily includes three isoforms, namely; PHD1, PHD2, and PHD3, of which PHD2 is the most abundantly expressed mammalian isoform. In normoxia, HIF-1a is hydroxylated by PHD, the cellular oxygen "sensor" that mediates oxygen-dependent hydroxylation of proline residue of HIF1a leading to subsequent ubiquitination and proteasomal degradation of HIF-1a [1]. In hypoxic situations, PHD activity is inhibited leading to stabilization of HIF-1 $\alpha$ in the nucleus and activation of transcription by the non-hydroxylated and stabilized form of HIF-1 $\alpha$, and finally, the expression of target genes which mediate its effects. The importance of PHD regulation of HIF-1a was shown in many studies that demonstrated that such regulation participates in the control of cardiorenal function and blood pressure [2-4].

Since the recognition that CVDs are intimately related to hypoxia, the HIF-1 $\alpha /$ PHD pathway has been evaluated as an important therapeutic target in the management of CVDs. Indeed, mechanisms sensing hypoxia may be important contributors to the development of pulmonary hypertension and to tissue injury and repair associated with hypertensive disease and in integrated aspects of cardiovascular function [5]. In this regard, hypoxia mimetic agents which inhibit PHD and increase HIF1 $\alpha$ expression and activity have been reported to attenuate CVDs and the attendant pathology in many experimental animal models of diseases including renal ischemic injury [6], myocardial I/R injury [7], and cerebral ischemia [8]. Similarly, PHD inhibition upregulated HIF-1 $\alpha$ level and attenuated salt-induced hypertension in Dahl S rats, a salt-sensitive hypertensive model [4]. These and other studies showing that PHD inhibition downregulated vascular angiotensin type 1 receptor [9], support a protective role for increased HIF-1 $\alpha$ expression and activity in CVDs. However, other studies documented the contrary: that hypoxia participates in the progression of CVDs. This is evidenced by the observation that HIFla was upregulated in DOCA/salt hypertension [10] and that HIF-1a induction promoted the pathology of AII-induced perivascular fibrosis [11] and neointimal formation in response to carotid artery injury [12]. This is underscored by the fact that hypoxia has been reported to elicit many effects including activation of circulating and local renin angiotensin system (RAS) [13,14]. However, in another study,

*Corresponding author: AAdebayo O Oyekan, Center for Cardiovascular Diseases, Texas Southern University, Houston, TX 77004, USA, Tel: 313 4258/4341; Fax: 713313 4342; E-mail: Oyekan_AO@TSU.EDU

Received August 11, 2014; Accepted October 01, 2014; Published October 06 , 2014

Citation: Dallatu MK, Nwokocha E, AGU N, Myung C, Newaz MA, ei al. (2014) The Role of Hypoxia-Inducible Factor/Prolyl Hydroxylation Pathway in Deoxycorticosterone Acetate/Salt Hypertension in the Rat. J Hypertens 3: 184. doi:10.4172/2167-1095.1000184

Copyright: (c) 2014 Dallatu MK, et al. This is an open-access article distributed under the terms of the Creative Commons Attribution License, which permits unrestricted use, distribution, and reproduction in any medium, provided the original author and source are credited. 
overexpression of PHD i.e. reduced HIF-1a in the kidneys of DOCA/ salt hypertensive rat led to a reduction in the pathological changes that are characteristic of this model of hypertension [15]. Unlike the high renin models of hypertension in which a definitive role for HIF/PHD system has been documented $[12,13]$, there is paucity of information on the DOCA/salt model, a low renin model of hypertension. In this study, we tested the hypothesis that increased HIF-1a consequent to reduced PHD expression contributes to DOCA/salt hypertensive renal injury and that PHD inhibition ameliorates this effect. In view of the role of reactive oxygen species (ROS) in DOCA/salt hypertension [16] and of nitric oxide in the effects associated with PHD inhibitors $[2,17]$, we also evaluated the possible involvement of nitric oxide and ROS in these effects. Our data showed reduced expression of PHD2 and increased expression of HIF-1 $\alpha$ in DOCA/salt hypertension that appears to be a defense mechanism against excessive hypertensive renal injury. Our data also demonstrated that PHD inhibition exerts a protective effect in this model of hypertension that appears to involve increased production of nitric oxide and/or reduced production of ROS.

\section{Materials and Methods}

\section{Ethical approval}

This study was approved by the Institutional Animal Care and Use Committee (IACUC) of Texas Southern University (TSU) and conforms to the institutional guidelines on animal care and use. The TSU's IACUC is accredited by the American Association for the Accreditation of Laboratory Animal Care and meets the National Institutes of Health standards as set forth in the "A Guide for Care and Use of Laboratory Animals" (DHHS publication [NIH] 8523, revised).

Unless specified otherwise, all chemicals were obtained from Sigma-Aldrich (St Louis, MO) and are of the highest analytical grade. Dimethyl oxallyl glycine (DMOG, Cayman Chemical Co, Ann Arbor, MI, USA), is a cell-permeable, competitive inhibitor of prolyl hydroxylase domain-containing proteins (PHD) leading to stabilization of HIF.

This study was carried out in young (4-5 week old) male and female rats (125-155 g Sprague-Dawley; Harlan Sprague-Dawley, Houston, TX, USA). In these experiments, all animals were uninephrectomized (UNx) and randomly divided into groups that received mineral oil (vehicle, $1 \mathrm{ml} / \mathrm{kg}$ s.c.) and tap water ad libitum (Control, $\mathrm{n}=6-9$ ), DOCA $(25 \mathrm{mg} / \mathrm{kg}$ s.c. twice weekly) and $1 \% \mathrm{NaCl}$ in their drinking water ad libitum (DOCA, $\mathrm{n}=6-9)$, DOCA and $1 \% \mathrm{NaCl}+\mathrm{DMOG}(15 \mathrm{mg} / \mathrm{kg}$ i.p. daily, $n=5-6$ ). The dose of DMOG was that reported in the literature to inhibit PHD [17]. An additional group of rats consisted of $\mathrm{UNx}$ animals placed on tap water ad libitum and treated with DMOG (15 $\mathrm{mg} / \mathrm{kg}$ i.p. daily, $\mathrm{n}=5-6$ ) alone. These animals were placed in metabolic cages once weekly for $24 \mathrm{hr}$ urine collection for biochemical analyses of sodium $\left(\mathrm{U}_{\mathrm{N}} \mathrm{V}\right)$ or protein $\left(\mathrm{U}_{\text {prot }} \mathrm{V}\right)$. At the end of three weeks, rats were anesthetized with thiobutabarbital (Inactin, $100 \mathrm{mg} / \mathrm{kg}$ i.p) and blood was collected from the carotid artery for determination of serum nitrite, creatinine, and 8-Isoprostane. Thereafter, a laparotomy was performed and kidneys were harvested from anaesthetized (pentobarbital sodium, $100 \mathrm{mg} / \mathrm{kg}$ i.p.; $\mathrm{n}=5-9$ per group) rats, weighed immediately and snap frozen in liquid nitrogen for immunoblotting experiments.

\section{Blood pressure measurement}

In another set of UNx animals ( $\mathrm{n}=5$ per group), radiotelemeters (PA11C40, Data Sciences International, St Paul, Minnesota, USA) were placed in the abdominal aorta under sodium pentobarbital $[50 \mathrm{mg} / \mathrm{kg}$; intraperitoneally (i.p.)] for continuous monitoring of blood pressure in conscious animals (following the manufacturer's protocol). The animals were allowed to recover from surgery and were stabilized for 1 week before starting the experiments. Treatment consisted of mineral oil $(1 \mathrm{ml} / \mathrm{kg}$ i.p; $\mathrm{n}=5)$, DOCA $+1 \% \mathrm{NaCl}(\mathrm{n}=5), \mathrm{DOCA}+1 \% \mathrm{NaCl}+$ DMOG $(n=5)$, or DMOG $(n=5)$ using doses as quoted in the groups above.

\section{Biochemical analyses}

Urinary protein $\left(\mathrm{U}_{\text {Prot }} \mathrm{V}\right)$ was determined by a colorimetric kit from Sigma-Aldrich (St Louis, MO, USA) and or serum nitrite was determined spectrophotometrically using the Griess assay [18]. Serum creatinine was determined colorimetrically using Jaffe method and serum 8-Isoprostane was determined by a colorimetric kit from Cell Biolabs Inc. (San Diego, CA).

\section{Immunoblotting of protein expression}

$40 \mu \mathrm{g}$ of protein from kidney homogenate was electrophoresed on $10 \%$ polyacrylamide gels and transferred to PVDF membrane (Amersham Pharmacia, NJ, USA). Blots were probed with rabbit polyclonal antibody to nephrin, PHD2, HIF-1a (Santa Cruz Biotechnology, Santa Cruz, CA, USA), KIM-1 (R\&D Systems, Inc., Minneapolis, MN, USA), and $\beta$ actin (Abcam, Cambridge, MA, USA) at 1:300 dilution, followed by incubation with appropriate horseradish peroxidase conjugated secondary antibodies at 1:8000 dilution. Immunocomplexes were visualized using an enhanced chemiluminescence (ECL-Plus) detection system from Amersham Pharmacia. The intensity of the bands was scanned and quantified using personal densitometer SI scanner and Image Quant analysis software (Molecular Dynamics, Sunnyvale, CA).

\section{Histopathologic studies}

Kidneys harvested from anaesthetized (pentobarbital sodium, $100 \mathrm{mg} / \mathrm{kg}$ i.p.; $\mathrm{n}=4-5$ per group) rats were subjected to formalin fixation and paraffin embedding. $4-\mu \mathrm{m}$ tissue sections were prepared and stained with hematoxylin and eosin (H\&E) and periodic acidSchiff (PAS) stains. Histological analysis was done single blind using a semiquantitative scoring method as described by Raij et al. [19]. All lesions were scored as follows based on the extent and severity: normal, grade 0 ; mild, grade 1 (involving up to $25 \%$ ); moderate, grade 2 (involving 25-50\%); severe, grade 3 (involving 50-75\%); very severe, grade 4 (involving $75-100 \%$ ), Medial thickening in small arteries and arterioles were scored as fold increases as follows: normal, 1; mild thickening, 1.5; moderate, 2.5; marked, 3.5 .

Collagen I staining: Immunohistochemical staining for collagen type I was performed to evaluate interstitial fibrosis. Four-micron paraffin-embedded tissue sections were submitted to immunoperoxidase staining using goat anti-rat polyclonal antibody against collagen type I (Southern Biotech, Birmingham, Alabama; dilution 1: 100) followed by peroxidase-labeled rabbit anti-goat polyclonal antibody (Dako, Carpinteria, California; Dilution 1:200). The severity of interstitial fibrosis was evaluated as the percentage of renal cortical tissue stained positive for collagen I. This was derived from the ratio of the grid points located within the areas of collagen deposition to the total number of grid points. These points were chosen randomly from 20 high powers (x 400) cortical fields and examined under a $1 \times 1 \mathrm{~cm}$ reticule with 121 grid points.

\section{Statistical Analysis}

Values were presented as mean \pm SEM. Means were compared 
Citation: Dallatu MK, Nwokocha E, AGU N, Myung C, Newaz MA, ei al. (2014) The Role of Hypoxia-Inducible Factor/Prolyl Hydroxylation Pathway in Deoxycorticosterone Acetate/Salt Hypertension in the Rat. J Hypertens 3: 184. doi:10.4172/2167-1095.1000184

Page 3 of 7

between groups and between treatments for significant difference using ANOVA followed by a Newman-Keul's multiple range test for comparisons within the groups and unpaired t-test for comparisons between groups. In all cases, $\mathrm{p}<0.05$ was considered significant.

\section{Results}

\section{Blood pressure elevation in DOCA/salt-treated rats - effect} of PHD inhibition

Figure 1a illustrates the changes in mean arterial blood pressure in animals placed on three weeks of treatment with DOCA and $1 \% \mathrm{NaCl}$ in the presence or absence of DMOG. Animals treated with DMOG alone (DMOG, $n=5$ ) did not show a change in blood pressure when compared to control $(n=5)$ rats. However, arterial blood pressure increased by $44 \pm 3 \%(\mathrm{P}<0.05 ; \mathrm{n}=5)$ in rats treated with $\mathrm{DOCA} / 1 \%$ $\mathrm{NaCl}$. On its own, DMOG did not affect blood pressure but it markedly blunted the increase in blood pressure in animals treated with DOCA (DOCA+DMOG, $P<0.05 ; n=5$ ). The inhibition by DMOG was evident as early as Day 7 but was most pronounced on Day 21.

\section{Renal injury following DOCA/1\% $\mathrm{NaCl}$ administration - effect of PHD inhibition}

The role of PHD/HIF-1a on kidney injury in animals placed on DOCA $/ 1 \% \mathrm{NaCl}$ (DOCA; $\mathrm{n}=9$ ) is illustrated in Figure 1b. Protein excretion was barely detectable in all the animals on Day 0 (baseline) of the study and there was no increase in protein excretion by Week 3 in control rats $(\mathrm{n}=8)$ or in uninephrectomized rats treated with DMOG alone (DMOG; $\mathrm{n}=5)$. However, protein excretion increased $(\mathrm{P}<0.05)$ as early as Week 1 in animals placed on DOCA/1\% NaCl, reaching a several-fold increase $(\mathrm{P}<0.01)$ by Week 3 . DMOG markedly blunted the proteinuria $(2.9$-fold; $\mathrm{P}<0.01)$ at Week 3 in animals placed on DOCA $/ 1 \% \mathrm{NaCl}$ (DOCA+ DMOG; $\mathrm{n}=6$ ).

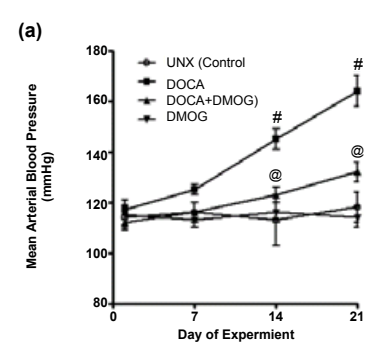

(c)

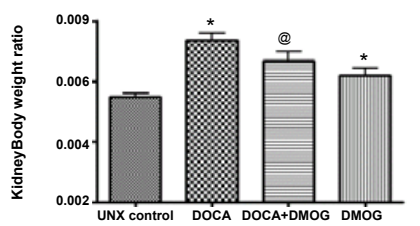

(b)

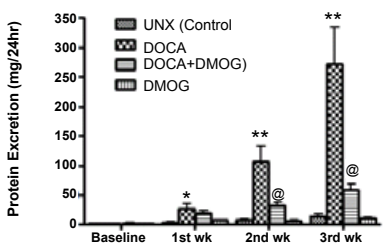

(d)

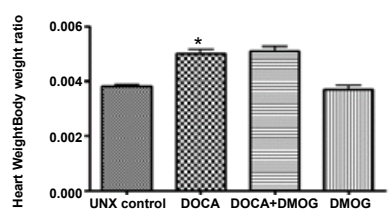

Figure 1: Mean arterial blood pressure (a), proteinuria (b), kidney weight- (c) and left ventricular/body weight indices (d) in uninephrectomized rats placed on $1 \% \mathrm{NaCl}$ (CONTROL, $\mathrm{n}=8$ ) treated with DOCA $(25 \mathrm{mg} / \mathrm{kg}$ s.c. twice weekly) in the absence (DOCA, $n=9)$ or presence of DMOG (15 mg/kg i.p; DOCA+ DMOG; $\mathrm{n}=6$ ). Animals that underwent uninephrectomy and received ordinary tap water were treated with DMOG (DMOG, $n=5$ ) for comparison.

$\# \mathrm{P}<0.05$ versus Control @P $<0.05$ vs DOCA

\#\#P<0.01) versus Control @@P<0.05 versus DOCA

Figure 1a represents data from 5 animals per group. The other $n$ values apply to Figure $1 \mathrm{~b}, \mathrm{c}$, and $\mathrm{d}$.

\section{Organ enlargement in DOCA/salt-treated rats - effect of PHD inhibition}

The effect of alteration of PHD/HIF-1a on organ hypertrophy in animals after three weeks of treatment with DOCA/ $1 \% \mathrm{NaCl}$ is illustrated in Figure 1c and d. Kidney/body weight increased markedly $(56 \pm 4 \%, \mathrm{P}<0.05 ; \mathrm{n}=9)$ in rats made hypertensive by treatment with DOCA/1\% NaCl. Kidney/body weight index increased slightly but significantly in uninephrectomized DMOG-treated $(17 \pm 3 \%, n=5)$ rats when compared to the untreated uninephrectomized controls $(\mathrm{P}<0.05)$. However, in animals treated with DOCA/1\% NaCl, DMOG reduced the renal hypertrophy $(\mathrm{P}<0.05 ; \mathrm{n}=6)$, to a level that is not significantly different from that seen in uninephrectomized DMOGtreated rats. Similarly, there was LV hypertrophy $(32 \pm 3 \%, \mathrm{P}<0.05)$ following three weeks of placement of rats on DOCA $/ 1 \% \mathrm{NaCl}(\mathrm{n}=9)$, Figure 1d. However, unlike the situation with kidney weight, DMOG did not affect LV hypertrophy in these hypertensive animals neither did it affect LV weight/body weight index in control animals. The lack of corresponding quantitative effects by DMOG on organ enlargement as with blood pressure elevation suggests that the effects produced by DMOG are independent of blood pressure changes.

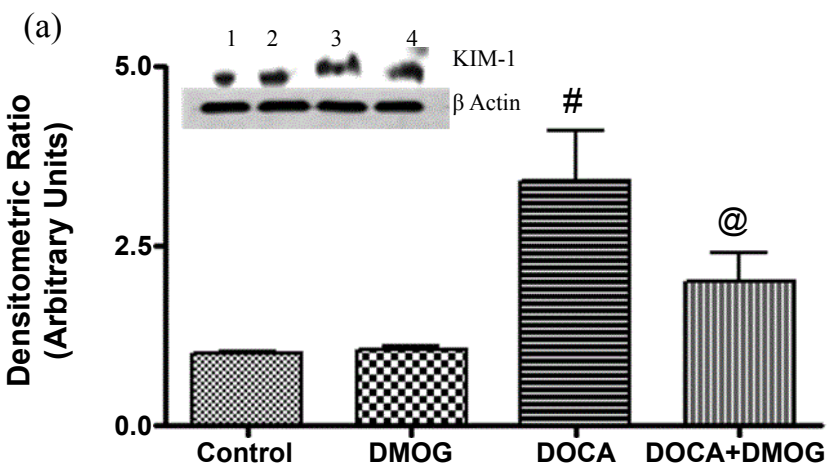

(b)

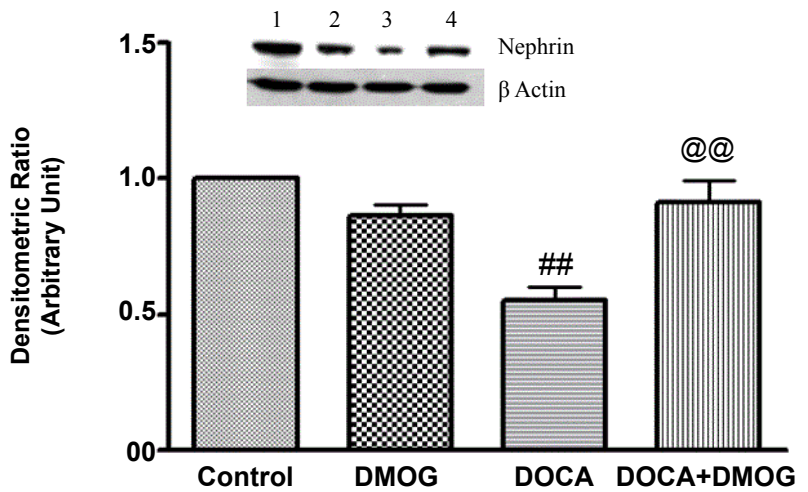

Figure 2: Expression of $\mathrm{KIM}-1$ (a) or nephrin (b) in whole kidneys of uninephrectomized rats placed on $1 \% \mathrm{NaCl}$ in drinking water (CONTROL, $n=6$; Lane 1) treated with DOCA ( $25 \mathrm{mg} / \mathrm{kg} \mathrm{s.c.} \mathrm{twice} \mathrm{weekly)} \mathrm{in} \mathrm{the} \mathrm{absence} \mathrm{(DOCA,}$ $n=9$; Lane 3) or presence of DMOG (DOCA+DMOG; $n=6$; Lane 4). A set of animals that underwent uninephrectomy were placed on tap water and treated with DMOG alone (DMOG; $n=5$; Lane 2). A representative blot of the expression of KIM-1 or nephrin is shown with internal loading control ( $\beta$-actin). Graphs depict the quantitation by densitometry.

\#P<0.05 (\#\# $<0.01)$ versus Control @P $<0.05$ (@@P<0.01) versus DOCA 
Citation: Dallatu MK, Nwokocha E, AGU N, Myung C, Newaz MA, ei al. (2014) The Role of Hypoxia-Inducible Factor/Prolyl Hydroxylation Pathway in Deoxycorticosterone Acetate/Salt Hypertension in the Rat. J Hypertens 3: 184. doi:10.4172/2167-1095.1000184

Page 4 of 7

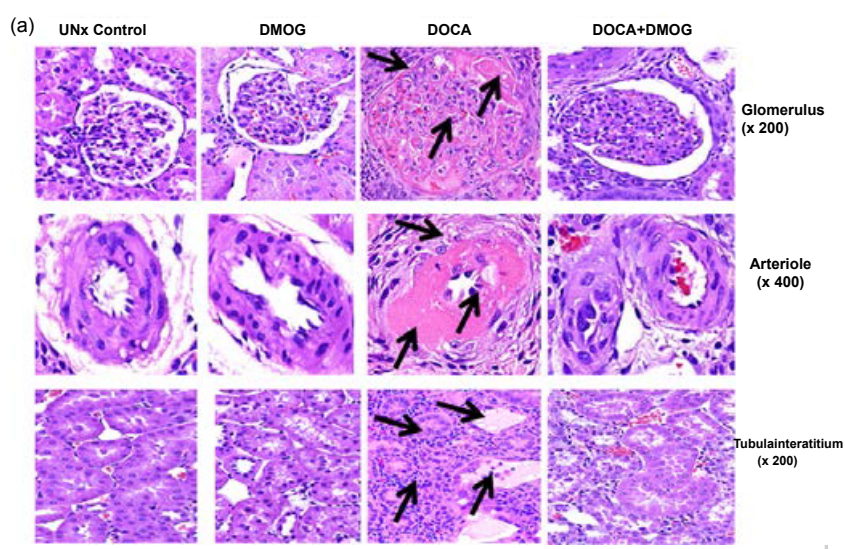

(b)
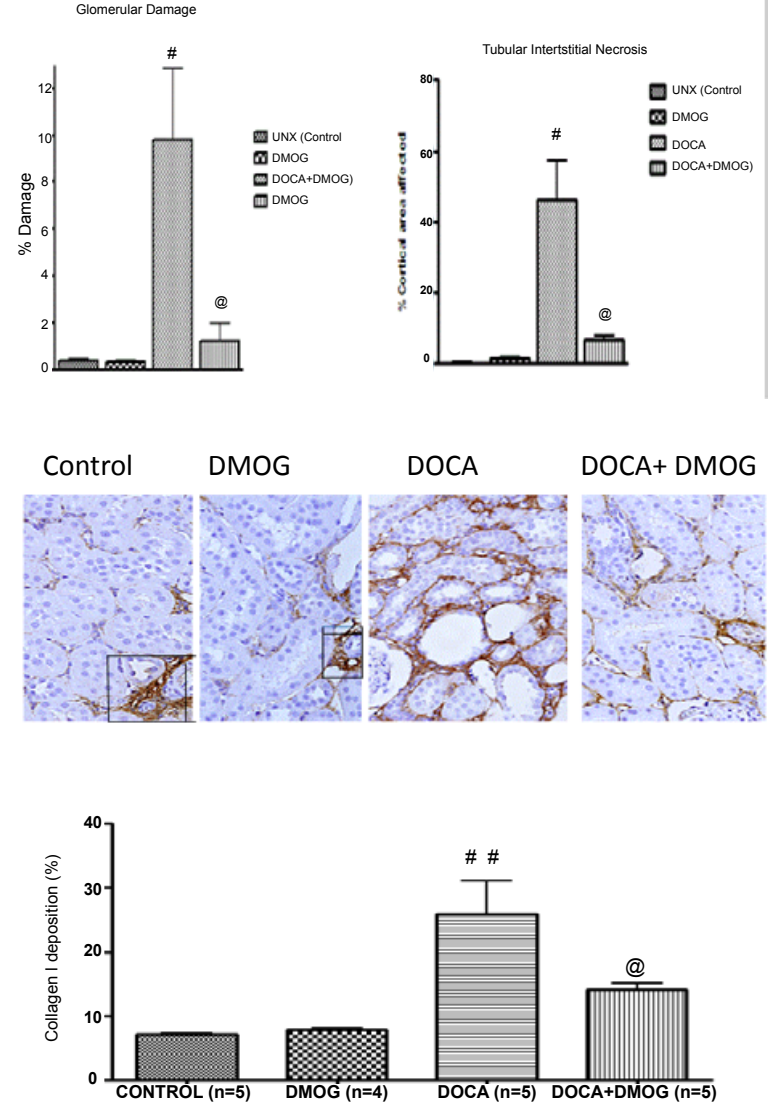

Figure 3: Histopathological changes $(a, b)$ or immunohistochemical staining for collagen I (c) in kidneys of uninephrectomized rats placed on $1 \% \mathrm{NaCl}$ in drinking water (CONTROL, $\mathrm{n}=8$ ) treated with DOCA ( $25 \mathrm{mg} / \mathrm{kg}$ s.c. twice weekly) in the absence (DOCA, $n=9$ ) or presence of DMOG (DOCA+ DMOG; $n=6$ ). A set of animals that underwent uninephrectomy were placed on tap water and treated with DMOG alone (DMOG; $n=6$ ).

Figure shows sections stained with hematoxylin and eosin ( $H$ \& E; a) or immunoperoxidase staining for collagen I (c). Magnifications are shown at x200 (x400 for the arteriole). Arrows show lesions in DOCA/salt hypertensive rats (DOCA) as described fully in the text: glomerular enlargement, hypercellularity, inflammatory infiltrates, fibrinoid deposition ( $a$, upper panel), thickening of arterial wall, periarterial fibrosis, mural disorganization (a, middle panel), interstitial inflammation, fibrosis, tubular atrophy or dilatation (a, lower panel). Quantitation of glomerular and tubular lesions are shown in Figure $3 \mathrm{~b}$ and that of collagen deposition are shown in Figure 3c. Normal deposition of collagen I (in rectangle inset).

\#P< 0.05 versus Control @ $\mathrm{P}<0.05$ versus DOCA

\section{Effect of PHD inhibition on markers of renal injury in DOCA/ salt hypertensive animals}

Figure 2a illustrates that accompanying the proteinuria at 3 weeks in rats made hypertensive by administration of DOCA and $1 \% \mathrm{NaCl}$ (DOCA; $\mathrm{n}=9$ ) was a 3.4-fold increase $(\mathrm{P}<0.01)$ in the expression of KIM1 , a marker of tubular injury. DMOG did not affect KIM-1 expression on its own (DMOG; $n=6$ ) but markedly blunted DOCA/salt-induced increase in KIM-1 expression. Similarly, in DOCA/salt-hypertensive rats, there was a marked reduction $(61 \pm 4 \%$; $\mathrm{P}<0.05)$ in the expression of nephrin, a marker of glomerular injury, Figure $2 \mathrm{~b}$. However, in the presence of DMOG (DOCA+DMOG; $n=6$ ), the decrease in nephrin expression was prevented $(\mathrm{P}<0.01)$. When compared to control rats, DMOG on its own (DMOG; $\mathrm{n}=5$ ) did not significantly affect nephrin expression.

\section{Histopathological changes in the kidney following DOCA/ salt treatment - Effect of DMOG}

Figure 3a illustrates representative glomerular (upper panel), arterial/arteriolar (middle panel), and tubulointerstitial lesions (lower panel) in rats placed on DOCA and $1 \% \mathrm{NaCl}$ (DOCA). Glomerularlesions consisted of glomerular enlargement, hypercellularity, inflammatory cell infiltration, and fibrinoid deposit (upper panel). Arterial changes consisted of fibrinoid deposit, destruction of arterial wall, medial thickening with periarterial fibrosis, reactive atypia of endothelial cells, and mural disorganization (middle panel). Tubulointerstitial lesions consisted of tubular interstitial fibrosis, mononuclear inflammatory cell infiltration, tubular atrophy and dilatation, loss of tubular segment differentiation and perivascular deposition of collagen (lower panel). In DOCA/salt hypertensive rats treated with DMOG (DOCA+DMOG; $\mathrm{n}=6$ ), these pathological changes were abolished or mild in extent and severity when compared to that observed in the DOCA/salt group. Figure $3 \mathrm{~b}$ illustrates glomerular damage and tubulointerstitial changes in quantitative terms.

Figure $3 c$ (upper panel) illustrates that immunoperoxidase staining for collagen I, though normally present in the kidney, is inconspicuous in control and DMOG-treated rats (DMOG) but perivascular and interstitial deposition is marked and diffuse in DOCA/salt hypertensive rats (DOCA, $n=5$ ). In DOCA/salt hypertensive rats that were treated simultaneously with DMOG (DOCA+DMOG, $n=5$ ), collagen I deposition is markedly attenuated. Figure 3c (lower panel) is a quantitative illustration of collagen I staining, a marker of fibrosis, in these animals. Collagen I staining was about $5 \%$ in the control $(n=5)$ and DMOG-treated $(n=4)$ rats but the staining increased 3 -fold $(\mathrm{P}<0.01)$ in DOCA/salt hypertensive $(\mathrm{n}=5)$ rats and this was markedly blunted $(\mathrm{P}<0.05)$ by DMOG.

\section{Effect of DMOG on PHD2 and HIF-1a expression in DOCA/ salt hypertension}

Figure 4a illustrates that there was reduced PHD2 protein expression $(43 \pm 6 \%$; $\mathrm{P}<0.01)$ in rats placed on $\mathrm{DOCA} / 1 \% \mathrm{NaCl}$ treatment for 3 weeks (DOCA; $n=9$ ). In DOCA/salt-treated rats that were treated additionally with DMOG (DOCA+ DMOG; $n=6$ ), PHD2 protein expression was unchanged $(37 \pm 4 \%$ : $\mathrm{P}<0.05)$ from that seen in the rats placed on DOCA/salt alone. When compared to uninephrectomized rats placed on tap water (Control, $n=8$ ), DMOG on its own, reduced PHD protein expression $(23 \pm 3 \%, \mathrm{P}<0.05 ; \mathrm{n}=5)$; an effect that is consistent with its widely recognized effect as a PHD inhibitor. By contrast, HIF-1a protein expression was increased (47 $\pm 5 \%, \mathrm{P}<0.05)$ in rats treated with DOCA/salt for 3 weeks, Figure $4 \mathrm{~b}$. 
(a)

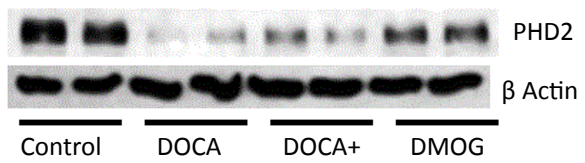

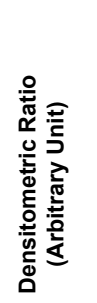

(b)
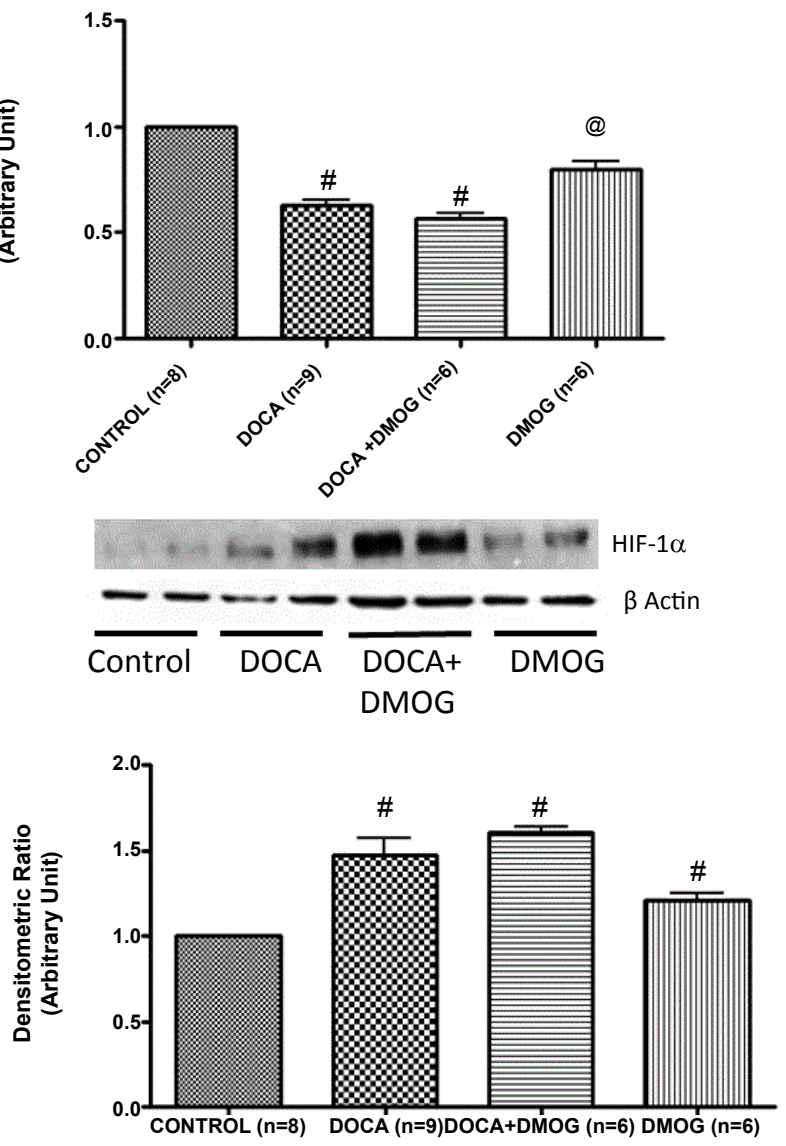

Figure 4: PHD2 (a) or HIF-1 $\alpha$ (b) expression in whole kidneys of uninephrectomized rats placed on $1 \% \mathrm{NaCl}$ in drinking water (CONTROL, $n=8$ ) treated with DOCA (25 mg/kg s.c. twice weekly) in the absence (DOCA, $n=9$ ) or presence of DMOG (DOCA+ DMOG; $n=6$ ). A set of animals that underwent uninephrectomy were placed on tap water and treated with DMOG alone (DMOG; $n=5$ ). A representative blot of the expression of PHD2 or HIF-1 $\alpha$ is also shown with internal loading control ( $\beta$-actin). Graphs depict quantitation of immunoblot by densitometry.

\#P $<0.05$ versus Control @ $\mathrm{P}<0.05$ versus DOCA

Treatment with DMOG did not affect the increase in HIF-1a protein expression in DOCA/salt-treated animals. However, consistent with its negative regulator effect on HIF- $1 \alpha$, DMOG alone increased basal HIF$1 \alpha$ protein expression $(23 \pm 3 \% ; \mathrm{P}<0.05)$.

Effect of DMOG on renal function, nitric oxide and reactive oxygen species production in DOCA/salt hypertensive animals

Figure 5a illustrates serum creatinine, an index of renal function, or serum nitrite, an index of systemic NO availability, or 8-isoprostane, an index of free radical production in animals placed on DOCA $/ 1 \% \mathrm{NaCl}$ in the absence or presence of DMOG. There was more than a 2 -fold increase $(\mathrm{P}<0.05)$ in serum creatinine after three weeks of treatment with DOCA $/ 1 \% \mathrm{NaCl}(\mathrm{n}=6)$ when compared to uninephrectomized controls $(n=6)$. DMOG treatment (DOCA+DMOG; $n=6)$ blunted
DOCA/salt-induced increase in serum creatinine by $50 \pm 7 \%$; $<<0.05$ ). On its own, DMOG did not affect serum creatinine as the level was not different from that in uninephrectomized controls. Figure $5 \mathrm{~b}$ illustrates that compared to uninephrectomized controls $(n=6)$, serum nitrite decreased markedly $(57 \pm 6 \%, \mathrm{P}<0.05)$ after three weeks of treatment with DOCA and $1 \% \mathrm{NaCl}$. DMOG, on its own (DMOG; $n=5$ ) tended to increase serum nitrite. However, DMOG abolished the reduction in serum nitrite in rats treated with DOCA and $1 \% \mathrm{NaCl}$ reversing it completely. Coincident with these changes, serum 8-isoprostane level was higher $(72 \pm 7 \%, \mathrm{P}<0.05)$ in $\mathrm{DOCA} /$ salt hypertensive rats and DMOG virtually abolished this increase $(89 \pm 5 \%, \mathrm{P}<0.05)$.

\section{Discussion}

The HIF-1 1 /PHD system regulates cardiovascular function and CVDs is intimately linked to reduced oxygen-concentration state with inhibition of PHD recognized as a potential treatment for many CVDs. In this study, we report decreased PHD2 expression and increased HIF-1a expression, hypertensive renal injury, increased expression of KIM-1, an index of tubular injury, decreased expression of nephrin, an index of glomerular injury, and increased collagen I staining, an index of fibrosis in DOCA/salt hypertensive rats. In addition, there was

(a)

(b)
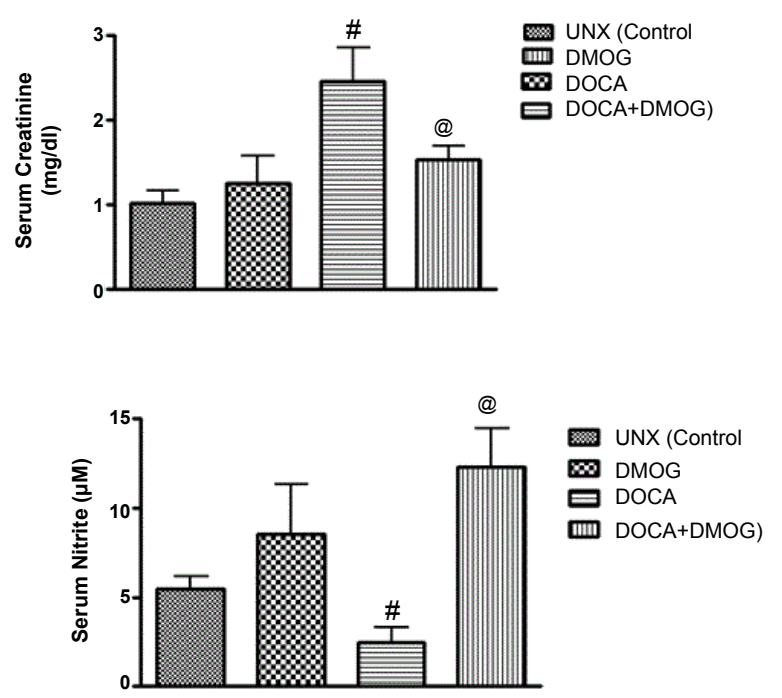

(c)

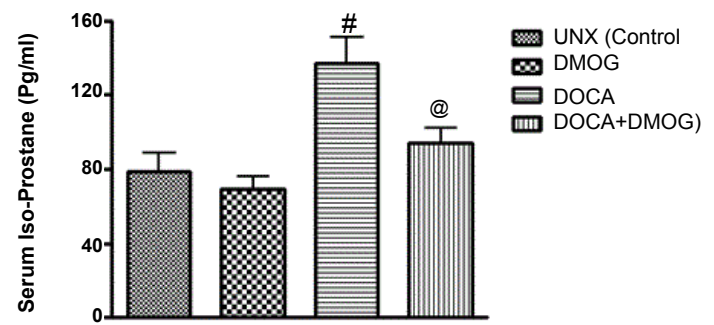

Figure 5: Serum creatinine (a), nitrite (b), or 8-Isoprostane (c) in rats after three weeks of treatment with $\mathrm{DOCA} / 1 \% \mathrm{NaCl}(\mathrm{DOCA}, \mathrm{n}=5)$ or with $\mathrm{DMOG}$ $(D O C A+D M O G, n=6)$. Serum levels in rats placed on tap water treated with DMOG alone (DMOG, $n=5$ ) or in uninephrectomized rats placed on $1 \% \mathrm{NaCl}$ drinking water (Control, $n=6$ ) are also shown.

\#P $<0.05$ versus Control @ $\mathrm{P}<0.05$ versus DOCA 
marked reduction in serum nitrite but higher serum creatinine and 8-Isoprostane alongside with histopathological changes that consisted of glomerular and fibrinoid changes, tubular interstitial necrosis, and mononuclear inflammatory cell infiltration among others. DMOG, a widely recognized PHD inhibitor [9,20], markedly blunted these effects and improved renal function. These data are consistent with studies that demonstrated protective effect of inhibition of PHD as was reported for AII-induced vascular remodeling in the mouse heart [11], podocyte injury and proteinuria in the remnant kidney [20] or gentamicin-induced nephrotoxicity [21].

Studies involving hypoxia/HIF- $1 \alpha$ and PHD and DOCA/ salt hypertension are few and far between but a major study [10] demonstrated that the initial phase following DOCA treatment was characterized by a reduction in the population of oxygen- and nutrientsupplying peritubular capillaries (PTCs) accompanied by elevation of HIF-1 $\alpha$ and VEGF, two hypoxia-responsive angiogenic factors, without affecting blood pressure or eliciting any pathology. There was a delayed phase during which HIF- $1 \alpha$ and VEGF expression was greatly enhanced alongside hypertensive renal damage and enhanced apoptosis. Data from the current study also demonstrated no change in blood pressure or any indication of renal damage during Week 1 of DOCA treatment -the period during which there was reduction in PTCs [10]. However, there was a downregulation of PHD2 and increased expression of HIF$1 \alpha$ at three weeks - a stage that corresponded to the delayed phase of DOCA/salt exposure characterized by renal pathology [10]. Based on these data, it appears that the potential sequence of events following DOCA/salt treatment is reduced oxygen tension, reduced PHD2 expression/activity, increased HIF-1a stabilization, continued hypoxia, hypertension and damage to kidney, heart, and vasculature.

The question arises whether PHD inhibition/HIF-1a especially at the latter stage of DOCA/salt hypertension will be counterproductive because of the potential to exacerbate the ongoing pathology. However, the notion is countered by the possibility that HIF-1 $\alpha$ stabilization at the latter stage of DOCA/salt treatment was necessary to mitigate against hypertensive renal damage and not to propagate it as demonstrated by the protective effect of PHD inhibition [11,20,21]. However, data from other studies revealed that the effect of PHD inhibition appears to depend, among other things, on the type of CVD and whether it is acute or chronic in nature. Thus, acutely injured kidneys appear to benefit from the protective effects of HIF-regulated biological processes, while chronic hypoxia, mediated in part through HIF-1a, can contribute to increased extracellular matrix production and thereby potentially promote the progression of renal disease [22]. Indeed, overexpression of PHD3 gene blunted renal injury in the DOCA/salt hypertensive rat [15] leading the authors to suggest that PHD inhibition and subsequent HIF-1 a stabilization may be an important pathway to hypertension and end organ damage under pathological conditions. This is not surprising considering that hypoxia attenuates renal function in hypertension [23] exacerbating the ongoing pathology.

In considering whether PHD inhibition/increased HIF-1a protects or promotes tissue/organ damage, we recognize that the conventional premise is that HIF-1a mitigates against the damaging effect of hypoxia by regulating the expression of genes vital for cellular adaptive responses to hypoxia such as glycolysis, erythrocytosis, and angiogenesis [3,9]. Thus, inhibition of PHD in hypoxia should result in stabilization and activation of HIF-1 $\alpha$ for adaptive (protective) effects on tissues [3,24]. Thus, in this study, DMOG protected against hypertensive renal injury in DOCA/salt hypertension suggesting that reduced expression of PHD2, consequent to increased HIF-1a expression/activity is a defense mechanism in DOCA/salt hypertension. The lack of additional effect by DMOG on PHD expression in DOCA/salt-treated rats despite its strong protective effects on hypertension and end organ damage may be accounted for by the possibility that PHD inhibition has a negligible effect on HIF-1 $\alpha$ activation as was reported in AII-induced vascular fibrosis [14]. Thus, PHD inhibition does not necessarily translate to a commensurate decrease in PHD expression or increase in HIF-1a expression. In fact, a recent study demonstrated a paradoxical increase in PHD3 by DMOG despite its renoprotective effect in the remnant kidney [20].

In evaluating the possible mechanism for the protective effect of DMOG in this study, we examined a role for nitric oxide. This is because (i) NO synthase is a target gene for HIF- $1 \alpha[1,7,17]$ and (ii) NO regulates PHD $[2,17]$ leading to attenuation of proline hydroxylation of HIF-1a. Indeed, in mice subjected to renal ischemia/reperfusion injury, DMOG up-regulated NOS expression [24]. In this study, the increased serum nitrite level in DMOG-treated rats and the blunting of the reduced serum nitrite in DOCA/salt hypertensive rats treated with DMOG is consistent with these observations, underscoring the possibility that NO production is a mechanism for PHD inhibitionrelated protection in DOCA/salt hypertension. Moreover, the improved renal function (reduced serum creatinine) in DMOG-treated rats; consistent with blunted KIM-1 expression and increased nephrin expression supports DMOG-related preservation of glomerular and tubular function in DOCA/salt hypertension, a low renin model. The relationship between hypoxia vis-à-vis the HIF/PHD system on renin is an ongoing debate. Hypoxia was shown to upregulate renin [13] but a recent study involving stabilization of HIF in mice through conditional deletion of von Hippel-Lindau (vHL) protein, its negative regulator, resulted in accumulation of HIF- $1 \alpha$ and $-2 \alpha$ but a reduction in expression of renin and number of renin-producing cells [25]. Though the jury is still out, the latter suggests that hypoxia-inducible genes are essential for normal development and physiologic adaptation of renin-producing cells. It therefore appears that the HIF/PHD system is relevant in low and high renin models as seen in the protective effect of PHD inhibition in AII-induced vascular inflammation [11] and the remnant kidney [20].

As DOCA/salt hypertension is characterized by increased vascular production of ROS [16] that is attributable to eNOS uncoupling [26] and reduced NO bioavailability [27], among others, we evaluated the effect of DMOG on oxidative stress. Our data revealed that the antihypertensive and renoprotective effects of DMOG may be accounted for by its ability to reduce production of reactive oxygen species through increased NO production. This is consistent with the established role of HIF in oxidative stress and the observation that DMOG-associated HIF-1a activation blunted gentamicin-induced nephrotoxicity and renal injury in the remnant kidney model through reduced oxidative stress and/or increase in antioxidant species $[20,21]$.

\section{Conclusion}

Data from this study suggest that reduced PHD2 expression and consequent increase in HIF-1 $\alpha$ expression probably result from hypoxia induced by DOCA/salt treatment. This priming event was accompanied by hypertension or renal damage at later stages when the continued hypoxia, reduced PHD2 expression and increased HIF-1a expression were accompanied by hypertensive renal injury and collagen deposition. Despite the lack of additional effect on PHD expression, a protective effect of a known PHD inhibitor in DOCA/salt hypertension emerges by mechanisms that are in part related to increase $\mathrm{NO}$ and/or reduced reactive oxygen species production. 
Citation: Dallatu MK, Nwokocha E, AGU N, Myung C, Newaz MA, ei al. (2014) The Role of Hypoxia-Inducible Factor/Prolyl Hydroxylation Pathway in Deoxycorticosterone Acetate/Salt Hypertension in the Rat. J Hypertens 3: 184. doi:10.4172/2167-1095.1000184

\section{Acknowledgement}

This study was supported by the National Institutes of Health grant HL03674. The facilities of the RCMI program at Texas Southern University were used for this study.

\section{References}

1. Huang LE, Gu J, Schau M, Bunn HF (1998) Regulation of hypoxia-inducible factor 1 alpha is mediated by an $\mathrm{O}_{2}$-dependent degradation domain via the ubiquitin-proteasome pathway. Proc Natl Acad Sci U S A 95: 7987-7992.

2. Li N, Yi F, dos Santos EA, Donley DK, Li PL (2007) Role of renal medullary heme oxygenase in the regulation of pressure natriuresis and arterial blood pressure. Hypertension 49: 148-154.

3. Wang Z, Zhu Q, Xia M, Li PL, Hinton SJ, et al. (2010) Hypoxia-inducible factor prolyl-hydroxylase 2 senses high-salt intake to increase hypoxia inducible factor 1alpha levels in the renal medulla. Hypertension 55: 1129-1136.

4. Zhu Q, Wang Z, Xia M, Li PL, Zhang F, et al. (2012) Overexpression of HIF$1 \hat{1} \pm$ transgene in the renal medulla attenuated salt sensitive hypertension in Dahl S rats. Biochim Biophys Acta 1822: 936-941.

5. Gupte SA, Wolin MS (2012) Relationships between vascular oxygen sensing mechanisms and hypertensive disease processes. Hypertension 60: 269-275.

6. Matsumoto M, Makino Y, Tanaka T, Tanaka H, Ishizaka N, et al. (2003) Induction of renoprotective gene expression by cobalt ameliorates ischemic injury of the kidney in rats. J Am Soc Nephrol 14: 1825-1832.

7. Xi L, Taher M, Yin C, Salloum F, Kukreja RC (2004) Cobalt chloride induces delayed cardiac preconditioning in mice through selective activation of HIF1 alpha and AP-1 and iNOS signaling. Am J Physiol Heart Circ Physiol 287 H2369-2375.

8. Bergeron M, Gidday JM, Yu AY, Semenza GL, Ferriero DM, et al. (2000) Role of hypoxia-inducible factor-1 in hypoxia-induced ischemic tolerance in neonatal rat brain. Ann Neurol 48: 285-296.

9. Matsuura H, Ichiki T, Ikeda J, Takeda K, Miyazaki R, et al. (2011) Inhibition of prolyl hydroxylase domain-containing protein downregulates vascular angiotensin II type 1 receptor. Hypertension 58: 386-393.

10. Iwazu Y, Muto S, Fujisawa G, Nakazawa E, Okada K, et al. (2008) Spironolactone suppresses peritubular capillary loss and prevents deoxycorticosterone acetate/salt-induced tubulointerstitial fibrosis. Hypertension 51: 749-754.

11. Zhao Q, Ishibashi M, Hiasa K, Tan C, Takeshita A, et al. (2004) Essential role of vascular endothelial growth factor in angiotensin II-induced vascular inflammation and remodeling. Hypertension 44: 264-270.

12. Karshovska E, Zernecke A, Sevilmis G, Millet A, Hristov M, et al. (2007) Expression of HIF-1alpha in injured arteries controls SDF-1alpha mediated neointima formation in apolipoprotein $\mathrm{E}$ deficient mice. Arterioscler Thromb Vasc Biol 27: 2540-2547.
13. Krämer BK, Ritthaler T, Schweda F, Kees F, Schricker K, et al. (1998) Effects of hypoxia on renin secretion and renal renin gene expression. Kidney Int Suppl 67: S155-158.

14. Hubloue I, Rondelet B, Kerbaul F, Biarent D, Milani GM, et al. (2004) Endogenous angiotensin II in the regulation of hypoxic pulmonary vasoconstriction in anaesthetized dogs. Crit Care 8: R163-171.

15. Zhu Q, Wang Z, Zhu M, Han W (2011) Overexpression of HIF-prolyl hydroxylase transgene in the kidney attenuated renal injury in DOCA/salt hypertensive rats. Hypertension 8: e33-e183.

16. Somers MJ, Mavromatis K, Galis ZS, Harrison DG (2000) Vascular superoxide production and vasomotor function in hypertension induced by deoxycorticosterone acetate-salt. Circulation 101: 1722-1728.

17. Callapina M, Zhou J, Schnitzer S, Metzen E, Lohr C, et al. (2005) Nitric oxide reverses desferrioxamine- and hypoxia-evoked HIF-1alpha accumulationimplications for prolyl hydroxylase activity and iron. Exp Cell Res 306: 274 284.

8. Deng A, Arndt MA, Satriano J, Singh P, Rieg T, et al. (2010) Renal protection in chronic kidney disease: hypoxia-inducible factor activation vs. angiotensin II blockade. Am J Physiol Renal Physiol 299: F1365-1373.

19. Raij L, Azar S, Keane W (1984) Mesangial immune injury, hypertension, and progressive glomerular damage in Dahl rats. Kidney Int 26: 137-143.

20. Song YR, You SJ, Lee YM, Chin HJ, Chae DW, et al. (2010) Activation of hypoxia-inducible factor attenuates renal injury in rat remnant kidney. Nephrol Dial Transplant 25: 77-85.

21. Ahn JM, You SJ, Lee YM, Oh SW, Ahn SY, et al. (2012) Hypoxia-inducible factor activation protects the kidney from gentamicin-induced acute injury. PLoS One 7: e48952.

22. Haase VH (2006) Hypoxia-inducible factors in the kidney. Am J Physiol Renal Physiol 291: F271-281.

23. Welch WJ, Baumgärtl H, Lübbers D, Wilcox CS (2001) Nephron pO2 and renal oxygen usage in the hypertensive rat kidney. Kidney Int 59: 230-237.

24. Zhang XL, Yan ZW, Sheng WW, Xiao J, Zhang ZX, et al. (2011) Activation of hypoxia-inducible factor-1 ameliorates postischemic renal injury via inducible nitric oxide synthase. Mol Cell Biochem 358: 287-295.

25. Kurt B, Paliege A, Willam C, Schwarzensteiner I, Schucht K, et al. (2013) Deletion of von Hippel-Lindau protein converts renin-producing cells into erythropoietin-producing cells. J Am Soc Nephrol 24: 433-444.

26. Landmesser U, Dikalov S, Price SR, McCann L, Fukai T, et al. (2003) Oxidation of tetrahydrobiopterin leads to uncoupling of endothelial cell nitric oxide synthase in hypertension. J Clin Invest 111: 1201-1209.

27. Youn JY, Wang T, Blair J, Laude KM, Oak JH, et al. (2012) Endotheliumspecific sepiapterin reductase deficiency in DOCA-salt hypertension. Am J Physiol Heart Circ Physiol 302: H2243-2249. 